\title{
Challenges of Integrating Tobacco Cessation Interventions in TB Programmes: Case Studies from Nepal and Pakistan
}

\author{
Omara Dogar, ${ }^{1}$ Helen Elsey, ${ }^{2}$ Sudeepa Khanal, ${ }^{3}$ and Kamran Siddiqi ${ }^{1}$ \\ ${ }^{1}$ Department of Health Sciences, University of York, Heslighton, York, UK \\ ${ }^{2}$ Nuffield Centre for International Health and Development, Leeds Institute of Health Sciences, University of Leeds, Leeds, UK \\ ${ }^{3}$ Health Research and Social Development Forum (HERD), Thapathali, Kathmandu, Nepal
}

\begin{abstract}
ntroduction: Offering tobacco cessation interventions to TB patients is highly desirable due to the interaction between TB and tobacco use and the potential benefits of quitting. However, implementing such interventions in TB programmes remains a challenge and an under-researched area. Using two initiatives to implement tobacco cessation within TB programmes in Nepal and Pakistan as case studies, we describe these challenges and highlight lessons learnt in the process.

Methods: We first conducted a documentary review of all published and unpublished reports of the two initiatives including relevant qualitative and quantitative data and its' analyses. We then analysed this information using the Consolidated Framework for Implementation Research (CFIR) under the five domains of implementation: intervention characteristics, outer settings, inner settings, participant characteristics and the process of implementation.

Findings: We faced a number of challenges in implementing tobacco cessation within TB programmes both in Nepal and in Pakistan. These included: doubts about the contextual relevance of the intervention, environments conducive to smoking and political inertia for a cultural shift, service providers' workload, priorities, and their motivation and capacity to deliver tobacco cessation, and inadequate training and support during implementation. We learned that by adapting intervention to the local context, securing mandate from higher authorities, aligning tasks to service providers' roles and receptivity, building capacity through adequate training and providing support, monitoring and feedback during implementation can help in integrating tobacco cessation within TB programmes.

Conclusions: Lessons from existing studies can help TB programmes in implementing tobacco cessation interventions and enable greater integration and sustainability of cessation services within routine TB care.
\end{abstract}

\section{Introduction}

Syndemic is a synergistic interaction between two or more health problems that occurs at multiple levels of causation, contributing to a population burden of disease greater than the individual burden (Littleton \& Park, 2009). TB and tobacco use is such a syndemic. These two global epidemics amplify each other's negative impact on population health. Tobacco smoking increases the risk of acquiring TB infection, developing disease and worsening outcomes, including death, treatment failure and relapse (Bates et al., 2007; Lin, Ezzati, \& Murray, 2007; Siddiqi \& Lee, 2009; Slama et al., 2007). In many low-and-middle income countries (LMICs), where TB burden is high, tobacco use is also increasing, thus driving these epidemics on a collision course (van Zyl Smit et al., 2010); invariably, it is the poorest and the vulnerable who are most affected (Lonnroth, Jaramillo, Williams, Dye, \& Raviglione, 2009).

Just as two health problems have synergistic adverse health impact, targeting one health problem within routine care provision for the other, multiplies its health benefits. Integrating tobacco cessation within TB programmes is such an initiative. Offering tobacco cessation in TB patients (Siddiqi et al., 2013) and those with other respiratory conditions (Elsey, Dogar, Ahluwalia, \& Siddiqi, 2015) can achieve higher quit success rates than general smokers. TB patients' anxiety due to fear of death and of infecting 
others and health care providers' close and frequent involvement in their care, maximises opportunities to influence patients' tobacco use, (Rice \& Stead, 2008; Whyte \& Kearney, 2003) often referred to as 'teachable moments' (McBride, Emmons, \& Lipkus, 2003). Moreover, the adverse health effects of smoking in TB patients (e.g. the immunological impairment) start to reverse within six weeks of stopping (Arcavi \& Benowitz, 2004). On wider benefits, very few LMICs have the basic infrastructure in place to offer tobacco cessation (Piné-Abata et al., 2013). While setting up cessation services could be costly, integrating tobacco cessation within TB programme is feasible and could be highly cost-effective (Siddiqi, 2014; West et al., 2015).

There is now sufficient evidence to suggest that tobacco cessation interventions are effective in general smokers (Lancaster \& Stead, 2005) as well as in those with TB (Awaisu et al., 2011; Campbell, Chaudhary, Holdsworth, \& Lyne, 2014; Louwagie, Okuyemi, \& Ayo-Yusuf, 2014; Sereno et al., 2012; Siddiquea et al., 2013). However, there is little evidence of whether these interventions translate in a meaningful way and work 'as well' in routine TB care settings, as they do in trials (Piné-Abata et al., 2013; Siddiqi, 2014). This is partly because of the underutilisation of implementation research designs and when used, it is often limited to process evaluation (Moore et al., 2015).

In the absence of relevant implementation science, the paper describes the challenges of implementing tobacco cessation within TB programme in LMICs by applying a framework approach on two examples of implementing tobacco cessation in routine TB care in Nepal and in Pakistan. We also highlight the key lessons learnt in the process.

\section{Methods}

We conducted a document review and analyses of all published and unpublished reports of the two case-studies including relevant qualitative and quantitative data and its' analyses (Bowen, 2009). We used framework approach to conduct content analysis of these documents (Bryman \& Burgess, 2002).

For Pakistan, qualitative studies were conducted at: pre-pilot (Jan-Mar 2009), post-pilot (Jun 2010) and posttrial (Jan-Mar 2012) stages. Pre-pilot qualitative study consisted of seven semi-structured focus group discussions (FGDs), four with TB care providers (doctors and paramedics/nurses) and three with TB patients, to inform intervention adaptation. The post-pilot qualitative study consisted of in-depth interviews with TB patients (6), TB care providers (4), district TB and tobacco authorities (2), to explore their experience regarding delivering the intervention messages, their form, cultural acceptability, timing in delivery, and other practicalities in understanding and delivering the tools. The post-trial qualitative study consisted of 12 semi-structured interviews with TB care providers (six with doctors, six with paramedics) and six FGDs with patients, at six selected trial sites, to document experiences regarding patient and system related barriers and facilitators of intervention implementation for proposing refinements. All interviews and FGDs were audio-recorded and transcribed verbatim for thematic analysis and quotes generation before report writing. Additionally, monitoring and supervision notes recorded in the Interim Technical Reports (generated every six months for the trial) were consulted.

The study in Nepal used qualitative and quantitative methods within an action research approach in three primary health care centres (PHCCs) in two districts of Nepal. Before implementation, 21 patient semi-structured interviews and two FGDs with health workers were conducted to inform the intervention design. During the six month implementation phase, two researchers facilitated action research meetings with staff and observed implementation, recording the process and their reflections in diaries. Patients were followed up three months after receiving the cessation intervention to determine tobacco use (verified biochemically with carbon monoxide measurement) and gain feedback on the intervention. A further five in-depth interviews with health workers and district managers provided reflections on the process and integration of the intervention within the health system.

Several theoretical frameworks are used to analyse intervention implementation; some focus on cluster Randomised Controlled Trial (RCT) designs (Akl, Treweek, Foy, Francis, \& Oxman, 2007; Grant, Treweek, Dreischulte, Foy, \& Guthrie, 2013), while others, like RE-AIM (Glasgow, McKay, Piette, \& Reynolds, 2001) based on Diffusion of Innovations Theory (Rogers, 2010), seek to explain innovations and their immersion in systems. These often do not have consistent terminologies. Moreover, the emphasis in these frameworks is usually on the extent of implementation and rarely on the processes. The CFIR provides a structural basis for translating effective health related interventions and is based on the following key theoretical domains of implementation (Damschroder et al., 2009): (1) intervention characteristics - consist of intervention source and stakeholders' perceptions regarding the quality of evidence of its effectiveness and the relative advantage of implementing it; (2) outer settings - include policy drivers, mandates, workforce incentives, priorities of the implementing body and strategies to promote the wider uptake of the intervention; (3) inner settings - involves organisational structure and culture including the compatibility of the intervention with health providers' values and workload; (4) participants characteristics - focus on provider's knowledge, skill, motivation and self-efficacy to achieve intended implementation and; (5) the process of implementation - involves the degree to which the development, training, implementation and feedback on its quality and process are carried out.

Document review was conducted by extracting data from the aforementioned studies and reports based on the CFIR as the coding framework. Key aspects from our 
findings have been categorised into the CFIR concepts presented above.

\section{Findings and Discussion}

We first describe the two case studies, and then present the challenges of implementing tobacco cessation in $\mathrm{TB}$ programme settings. Using the five domains described above, we critically appraise our findings in light of the scientific evidence and highlight the lessons learnt during implementation.

\section{Pakistan Study}

Action to Stop Smoking in Suspected Tuberculosis (ASSIST) in Pakistan was a pragmatic trial showing that behavioural support (BS) alone or in combination with bupropion was effective in promoting cessation in smokers with TB symptoms (Siddiqi et al., 2013). The behavioural intervention was based on the ' 5 As quit model' (World Health Organisation, 2001). The model relies on personal motivation to quit tobacco use. The BS comprised of information on the harms of smoking, benefits of stopping and identification and management of the social/psychological or environmental cues that trigger a smoker's desire to smoke and their withdrawal symptoms. With the help of an expert panel, it was adapted to the local context. Adaptation was informed by the findings of two FGDs with the TB care providers and clients, pilot testing of the materials and clients' feedback (Siddiqi, Khan, \& Ahmad, 2010). The adapted intervention was then delivered integrated within the National TB Programme (NTP) of Pakistan - a public health programme revived under the Ministry of Health to combat TB epidemic in the country (National TB Program, 2013). Embedded within the health infrastructure; the NTP clinics engaged in ASSIST trial were based at the rural, tehsil and district health facilities, with a catchment population ranging between 30,000 and 450,000. TB care providers (paramedics and nurses) that delivered BS assist in routine identification and reporting of new TB patients, educating them about TB and monitoring their treatment.

\section{Nepal Study}

The second case study is a mixed methods action research study conducted to assess challenges in implementing BS for tobacco cessation in three PHCCs in a rural and an urban district. Interviews with respiratory patients who smoked, FGDs with health workers and with senior decision makers within the Ministry of Health and Population informed the adaptation of the intervention. Brief advice was offered to smokers at their first consultation in the PHCC. Those motivated to quit attended a BS session, which used behaviour change techniques (Michie, Hyder, Walia, \& West, 2011) addressing five domains - motivation, attitude, skills and self-efficacy and social influences. These techniques included identifying triggers and cop- ing strategies, consideration of consequences to self and family and goal setting.

The intervention was delivered integrated within WHOs Practical Approach to Lung Health (PAL) which aims to improve management of respiratory conditions including TB. The programme is managed by NTP, Nepal and is integrated within PHCCs. The intervention was eventually extended to all patients in primary care. While it was senior health workers that provided brief advice, health workers who had training in PAL and were responsible for treating and supporting TB patients, provided BS.

\section{Challenges to Implement Tobacco Cessation within TB Programmes Intervention Characteristics}

Challenge 1 - Contextual relevance.

Lesson learnt - Adaptation of the intervention to the local context and pilot testing it to inform further cultural modifications before wider implementation.

Behavioural interventions, based on theories formulated and tested in high-income countries (HIC) can pose an implementation challenge in LMIC due to contextual differences. In ASSIST trial, a clear steer from the clients to include strong messages and imagery on the harms of tobacco use informed contextual adaptation of BS intervention (Pre-pilot qualitative study, Jan-Mar 2009). Expected to trigger contemplation, such strong messages were contrary to current practice in HICs, where there is a high level of awareness of the harms of tobacco use. Moreover, clients also quoted a number of misconceptions about tobacco use, which were also considered while adapting the intervention.

\footnotetext{
'There are these germs that cause craving [for cigarettes] inside ... and he starts smoking again'. [a client]

'If he doesn't (smoke), he would not be able to digest his food'. [a client]

Similarly, clients' perceptions of their personal risk due to tobacco use were also considered.

'I was given (smoking cessation) advice. I quit for six months, then saw that I was fine so (started smoking again)'. [a client]

'I went to doctor when I had a cough. The doctor asked me whether I smoked. He said, 'If you quit smoking, you will be fine'. I quit smoking for six months'. [a client]
}

Following adaptation, pilot testing of BS intervention enabled further modification to make it culturally appropriate before progressing to full implementation. Such piloting allowed providers to build experience and expertise in implementing a new intervention and to reflect on the modifications required before its wider application (Kitson, Harvey, \& McCormack, 1998; Rycroft-Malone et al., 2002). Following post-pilot qualitative study (Jun 2010) we included new images of women smoking hookah - a common form of smoking among women in rural Pakistan. Moreover, literacy issues also came up and were dealt 
by adding interactive elements such as thought provoking images and questions asked by the care provider.

Similarly, in Nepal, the preliminary preimplementation qualitative work (Sept-Oct 2012) highlighted many misconceptions about tobacco use, among both patients and health workers. The high prevalence of smokeless tobacco use came up in the discussions. The health workers knowledge of the health hazards of chewing tobacco was found to be very limited. As one health worker commented whilst chewing tobacco during a FGD:

'It is better when you chew tobacco, because you are not exposed to the smoke'. (Health Worker, male Phase 1, PHCC1)

BS materials and training were revised accordingly and health workers were reminded to inform patients to resist chewing as a substitute for cigarettes. The clients' interviews highlighted the stigma attached to women smoking, and as a result the pains they took to smoke in private locations with other women. In light of this, the materials were revised to include pictures of women smoking to reinforce the message that BS intervention was meant for both men and women.

\section{Outer Settings}

Challenge 2 - Environment conducive to smoking and political inertia for a cultural shift.

Lesson learnt - Foster a cultural shift within health system that is conducive to promote health and secure mandate from higher authorities.

Lack of cultural norms within a health system that are conducive to promote health can be a barrier to implement behaviour change interventions. In both case studies, we learned that a health system that lacks cultural norms such as 'patient-centred approach' or 'shared-decision making' (Légaré et al., 2014) deters providers in engaging in conversations about changing their clients' smoking behaviour.

Furthermore, partial or non-adherence to smoke free policies and visibility of smoking inside health facilities often by other healthcare providers was another barrier in promoting smoking cessation (pre-pilot qualitative study, Jan-Mar 2009). To promote smoke-free policies, 'smokefree signs' were posted in the health facilities in Pakistan. In Nepal, one of the PHCCs addressed this aspect by encouraging staff that smoked to try the intervention themselves. This proved highly effective, with the staff member not only reporting that he had quit, but also becoming an advocate for the intervention (researcher's reflective notes, implementation phase (October 2013-March 2014).

A supportive political environment is also an important factor in smooth implementation of new interventions. This often involves engaging regulatory bodies (governmental or other central entity) and securing external mandates or collaboration (Mendel, Meredith, Schoenbaum, Sherbourne, \& Wells, 2008). TB care providers in ASSIST trial expressed the need for authorisation from the Ministry of Health in order to provide legitimacy to their role as cessation advisors (pre-pilot qualitative study, Jan-Mar 2009). Subsequent meetings with the district health authorities resulted in securing a mandate to provide such services within healthcare facilities.

In Nepal, the passing of the Tobacco Product (Control and Regulation) Act, 2010 provided a conducive political environment to support implementation of the intervention (Government of Nepal, 2011). For example, there is an increased recognition in Ministry of Health and Population of the need to provide smoking cessation services to all the PHCC patients and not just respiratory patients. Despite the ambition of Ministry of Health and Population (MoHP), implementation of the smoking cessation services remains weak, particularly as it falls under the umbrella of PAL which is not well integrated in the routine health care system. However, from 2015, some of the indicators of smoking cessation have been included in the main OPD register (Government of Nepal - Ministry of Health and Population, 2014). This is a strong starting point for the integration of tobacco cessation in the mainstream services and further health system changes. The 'checklist effect' (Wyatt \& Spiegelhalter, 1991), where recording a patients' smoking status encourages the health worker to remember to ask a patient whether they smoke, may well enable this part of the intervention to become routine practice.

\section{Inner Settings}

Challenge 3 - Receptivity, workload, role alignment and priorities of the key stakeholders.

Lesson learnt -Choosing healthcare providers that are suited to the role, can prioritise, are receptive to the new intervention and are prepared to change their routine.

The health providers' receptivity to an intervention and the extent to which application of that intervention in their practice will be rewarded, supported and expected within their organisation contributes to its successful integration within routine care (Klein \& Sorra, 1996). TB care providers in ASSIST trial considered tobacco cessation advice as an important primary prevention message (pre-pilot qualitative study, Jan-Mar 2009). However, their receptivity of a new intervention varied, especially, if it meant deviation from their routine practice (post-trial qualitative study, Jan-Mar 2012).

Similarly in Nepal, while health workers recognised the importance of tobacco cessation, few saw this as part of their job and several expressed the need to be incentivised financially (post-implementation in-depth interviews May-August 2014):

\footnotetext{
'We can't just work like that without incentives because people do give their time and energy....... if there is no incentive then they may implement it for a day but after that no one will'. (Health worker, male)
}

Furthermore, the complexity of behavioural intervention and departure from routine practice for the TB care providers were some of the other impediments. 
Often the 'length' (number/duration of behavioural sessions) and 'breadth' (number of behaviour change techniques offered) determine the complexity of interventions (Kochevar \& Yano, 2006). These often require re-orientation and a clear departure from the existing practice (Greenhalgh, Robert, Macfarlane, Bate, \& Kyriakidou, 2004). FGDs, (pre-pilot qualitative study, Jan-Mar 2009) with the doctors and TB care providers in ASSIST trial, highlighted that doctors were reluctant to take on the additional tasks of assessing smoking status and deliver BS. In contrast, TB care providers were willing to offer cessation advice, as it appeared to fit with their role of educating patients on TB care. However, given their workload they could only take on one to two patients a day (post-pilot qualitative study, Jun 2010). Moreover, they expressed concerns (post-trial qualitative study, JanMar 2012) over the length and breadth of the intervention. As a solution, they suggested delivering the intervention in groups of two or three patients especially to prevent delays.

The perceived importance of implementing an intervention within an organisation (e.g. the NTP) is a significant predictor of implementation effectiveness (Helfrich, Weiner, McKinney, \& Minasian, 2007). When there is a strong organisational buy-in, providers regard implementation of that intervention as an important priority rather than a distraction from their 'real work' (Helfrich et al., 2007). During ASSIST trial, NTP was under pressure to roll out other TB specific initiatives e.g. Global Funds; priority was on $\mathrm{TB}$ disease and not on tobacco control. Furthermore, there was no mention of tobacco cessation advice in the routine TB care guidelines, in Pakistan (prepilot qualitative study, Jan-Mar 2009).

Similarly, NTP in Nepal did not adapt the tobacco cessation components of PAL to the local context. FGDs with health workers (pre-implementation qualitative work (Sept-Oct 2012) highlighted the limited implementation of PAL in practice. In particular, the limited on-going supervision had clearly undermined health workers motivation to implement PAL, including its tobacco cessation components:

'Initially we did well (with PAL) because there was a good supervision but later it got lost in between because there was no review'. (Health worker FGD PHCC1)

\section{Participant Characteristics}

Challenge 4 -Providers motivation, expertise and selfefficacy.

Lessons learnt - Train competent and motivated healthcare providers, and, ensure sustained support.

Health care providers' expertise in implementing the intervention (Klein \& Sorra, 1996) and their motivation to encourage behaviour change (Lennox et al., 1998) are some of the key considerations. While communicating and interacting with patients using flipbook resource was considered straight-forward and intuitive, an effective patient-provider interaction involved an emphasis on patient choice, reflective listening and tailoring advice accordingly. These base skills, which are part of a continuum of behaviour change, were difficult to acquire in shortterm and were found to be quite challenging both in Pakistan (post-trial qualitative study, Jan-Mar 2012) and in Nepal (researcher's reflective notes during implementation and post-implementation interviews Oct 2013-Aug 2014). The reflections of a district level manager highlight this issue:

'I have observed that very few health workers actually practice counselling.... But if the health worker just tells the patient; 'quit smoking or else you'll get cancer', this is not enough!' (District TB and Leprosy Officer, Urban District, Nepal)

Similarly, providers with self-efficacy in implementing behavioural support were likely to sustain delivery of the intervention and stay motivated in the face of arising challenges (Rimer \& Glanz, 2005). In ASSIST trial, TB care providers felt confident in their communication skills (pre-pilot qualitative study, Jan-Mar 2009). However, they stressed the need for doctors' involvement in initiating the advice to enhance clients' willingness to receive further support from TB care providers (pre-pilot qualitative study, Jan-Mar 2009). In Nepal, the respect patients held for the doctors providing the initial brief advice also clearly influenced their receptiveness to BS (post-implementation interviews May-August 2014):

'He spoke with confidence, very fluently and politely in such a social way that I felt it was easy to interact. I don't know whether he is a small or big doctor but he is great doctor for me. (Patient Interview Nepal)

Following training and support, some health workers had clearly been able to adequately support patients in their quit attempt:

'After counselling session, I felt more confident towards quitting. (Patient interview post-intervention Nepal)

In both studies, measures were taken to enhance selfefficacy, organise supportive supervision and secure collaboration and cooperation with other staff (Monitoring and Supervision notes-Interim Technical Report 4, Dec 2010).

\section{Process of Implementation}

Challenge 5 - Inadequate training and variations in practice.

Lesson learnt - Adequate training and support combined with monitoring and feedback.

Intervention delivery, as intended, is influenced by the degree of relevant training required by the health care provider (Lennox et al., 1998). Majority of the TB care providers (post-trial qualitative study, Jan-Mar 2012) felt that the training offered in ASSIST trial (one full day) was inadequate to learn behaviour change techniques. Furthermore, a need for refresher trainings to build their expertise was also highlighted. This training gap has also been observed in another study of tobacco cessation 
integration in TB programmes (Sereno et al., 2012). While the concept of behaviour change is easy to grasp, it is much more difficult to acquire skills to deliver behaviour change techniques (Hall, James, \& Roberts, 1997). Therefore, short and once-only training sessions might be insufficient for such interventions (Lennox et al., 1998; Sereno et al., 2012).

In the ASSIST trial, despite a structured practice manual outlining intervention delivery, varying intervention fidelity and cessation rates were observed in different TB clinics (Siddiqi et al., 2013). A follow-up study is currently examining how monitoring fidelity to intervention delivery can help to understand and reduce variations in the delivery and effect of the intervention.

In Nepal, FGDs with staff pre-intervention highlighted that even where training sessions lasted for several days, health workers struggled to retain the knowledge they had gained:

'We have taken so many trainings. We are trained for PAL as well and we have also received other trainings. But the thing is when we don't practice it every day, we tend to forget'. (Health worker male PHCC1)

Moving away from didactic, classroom style teaching to a more interactive approach where participants can act out patient-centred communication skills and behaviour change techniques in role plays, proved useful in Nepal.

It is important to strike a balance between providing sufficient training to understand key components and ensuring continual support during implementation. Our approach in Nepal allowed health workers to reflect on the methods they had tried that seemed particularly effective with different patients. The researchers played a valuable role in sharing these lessons across PHCCs to support other health workers to try these new techniques.

\section{Strengths and Limitations}

The two studies assessed here were by no means identical. One was a large randomised controlled trial and the other a small action-research study. While TB patients were included in the Nepal study, they were only one of the patient groups offered the intervention, unlike the Pakistan study which targeted only patients with TB symptoms. The difference may limit comparisons. However, the lessons learnt across the two contexts were very similar. Extensive reporting on the study proceedings and qualitative investigations, both before and during the implementation phases of the studies, were some of the strengths of our case studies.

\section{Conclusions}

We have learnt a number of lessons from these two case studies, including the need for a contextual adaptation of the intervention, fostering environments conducive to tobacco cessation, securing mandate from higher authorities, aligning tasks to service providers' roles and receptivity, building capacity through adequate training and providing support, monitoring and feedback throughout implementation. We have also shown that by using principles of implementation science, we could gain useful insight into barriers and facilitators to integrating services. Future researchers conducting implementation research in this area should also study issues of scalability and sustainability of tobacco cessation interventions within TB and HIV programmes. The health system challenges in Nepal and Pakistan are not unique and most of the barriers identified and lessons learnt in the above case studies are likely to be applicable to several LMIC facing syndemics such as TB and tobacco. The above findings are likely to benefit policy makers and practitioners facing such challenges and wish to learn how to best integrate services to address syndemics.

\section{Acknowledgements}

None.

\section{Financial Support}

This work received no specific grant from any funding agency, commercial or not-for-profit sectors. The case study from Pakistan (ASSIST trial) was funded by the International Development Research Centre (IDRC) and from Nepal was funded by UK aid from the UK government; however the views expressed do not necessarily reflect the UK government's official policies.

\section{Conflict of Interest}

None.

\section{Ethical Standards}

Ethical approval for this specific article was not required. However, the authors assert that all procedures undertaken to conduct the two studies (in Pakistan and Nepal) were approved by relevant national and institutional ethics committees on human experimentation and comply with the ethical standards of the Helsinki Declaration of 1975, as revised in 2008.

\section{References}

Akl, E. A., Treweek, S., Foy, R., Francis, J., \& Oxman, A. D. (2007). NorthStar, a support tool for the design and evaluation of quality improvement interventions in healthcare. Implementation Science, 2(1), 19.

Arcavi, L., \& Benowitz, N. L. (2004). Cigarette smoking and infection. Archives of Internal Medicine, 164(20), 2206.

Awaisu, A., Nik Mohamed, M. H., Mohamad Noordin, N., Abd Aziz, N., Syed Sulaiman, S. A., Muttalif, A. R. et al. (2011). The SCIDOTS project: Evidence of benefits of an integrated tobacco cessation intervention in tuberculosis care on treatment outcomes. Substance Abuse Treatment, Prevention, and Policy, 6, 26. Retrieved from http:// onlinelibrary.wiley.com/o/cochrane/clcentral/articles/668/ CN-00805668/frame.html. doi:10.1186/1747-597X-6-26. 
Bates, M. N., Khalakdina, A., Pai, M., Chang, L., Lessa, F., \& Smith, K. R. (2007). Risk of tuberculosis from exposure to tobacco smoke: A systematic review and meta-analysis. Archives of Internal Medicine, 167(4), 335.

Bowen, G. A. (2009). Document analysis as a qualitative research method. Qualitative Research Journal, 9(2), 2740.

Bryman, A., \& Burgess, B. (2002). Analyzing qualitative data. London and New York: Routledge, Taylor \& Francis Group.

Campbell, I., Chaudhary, R., Holdsworth, G., \& Lyne, O. (2014). Brief advice to tuberculosis patients in Nepal to stop smoking: A pilot study by the Britain Nepal medical trust. The International Journal of Tuberculosis and Lung Disease, 18(12), 1438-1442.

Damschroder, L. J., Aron, D. C., Keith, R. E., Kirsh, S. R., Alexander, J. A., \& Lowery, J. C. (2009). Fostering implementation of health services research findings into practice: A consolidated framework for advancing implementation science. Implement Sci, 4(1), 50.

Elsey, H., Dogar, O., Ahluwalia, J., \& Siddiqi, K. (2015). Predictors of cessation in smokers suspected of TB: Secondary analysis of data from a cluster randomized controlled trial. Drug and Alcohol Dependence, 155, 128-133.

Glasgow, R. E., McKay, H. G., Piette, J. D., \& Reynolds, K. D. (2001). The RE-AIM framework for evaluating interventions: What can it tell us about approaches to chronic illness management? Patient Education and Counseling, 44(2), 119127.

Government of Nepal- Ministry of Health and Population. (2014). Health Management Information System Indicators Book, Kathmandu, Nepal.

Government of Nepal. (2011). Tobacco product (control and regulation) act, 2010. Kathmandu: Constitutional Assembly.

Grant, A., Treweek, S., Dreischulte, T., Foy, R., \& Guthrie, B. (2013). Process evaluations for cluster-randomised trials of complex interventions: A proposed framework for design and reporting. Trials, 14(1), 15.

Greenhalgh, T., Robert, G., Macfarlane, F., Bate, P., \& Kyriakidou, O. (2004). Diffusion of innovations in service organizations: Systematic review and recommendations. Milbank Quarterly, 82(4), 581-629.

Hall, D., James, P., \& Roberts, S. (1997). Evaluation of training in behaviour change counselling skills: The application of clinical-audit methodology. Health Education Journal, 56(4), 393-403.

Helfrich, C. D., Weiner, B. J., McKinney, M. M., \& Minasian, L. (2007). Determinants of implementation effectiveness adapting a framework for complex innovations. Medical Care Research and Review, 64(3), 279-303.

Kitson, A., Harvey, G., \& McCormack, B. (1998). Enabling the implementation of evidence based practice: A conceptual framework. Quality in Health Care, 7(3), 149-158.

Klein, K. J., \& Sorra, J. S. (1996). The challenge of innovation implementation. Academy of Management Review, 21(4), 10551080 .
Kochevar, L. K., \& Yano, E. M. (2006). Understanding health care organization needs and context. Journal of General Internal Medicine, 21(S2), S25-S29.

Lancaster, T., \& Stead, L. (2005). Individual behavioural counselling for smoking cessation. Cochrane Database of Systematic Reviews, Issue 2. Art. No.: CD001292. DOI: 10.1002/14651858.CD001292.pub2.

Légaré, F., Stacey, D., Turcotte, S., Cossi, M. J., Kryworuchko, J., Graham, I. D. et al. (2014). Interventions for improving the adoption of shared decision making by healthcare professionals. Cochrane Database of Systematic Reviews, Issue 9. Art. No.: CD006732. DOI: 10.1002/14651858.CD006732.pub3.

Lennox, A. S., Bain, N., Taylor, R. J., McKie, L., Donnan, P. T., \& Groves, J. (1998). Stages of change training for opportunistic smoking intervention by the primary health care team. Part I: randomised controlled trial of the effect of training on patient smoking outcomes and health professional behaviour as recalled by patients. Health Education Journal, 57(2), 140-149.

Lin, H. H., Ezzati, M., \& Murray, M. (2007). Tobacco smoke, indoor air pollution and tuberculosis: A systematic review and meta-analysis. PLoS Medicine, 4(1), e20.

Littleton, J., \& Park, J. (2009). Tuberculosis and syndemics: Implications for Pacific health in New Zealand. Social Science \& Medicine, 69(11), 1674-1680.

Lonnroth, K., Jaramillo, E., Williams, B., Dye, C., \& Raviglione, M. (2009). Drivers of tuberculosis epidemics: The role of risk factors and social determinants. Soc Sci Med, 68, 2240-2246.

Louwagie, G., Okuyemi, K. S., \& Ayo-Yusuf, O. A. (2014). Efficacy of brief motivational interviewing on smoking cessation at tuberculosis clinics in Tshwane, South Africa: A randomized controlled trial. Addiction, 109(11), 1942-1952.

McBride, C., Emmons, K., \& Lipkus, I. (2003). Understanding the potential of teachable moments: The case of smoking cessation. Health Education Research, 18(2), 156-170.

Mendel, P., Meredith, L. S., Schoenbaum, M., Sherbourne, C. D., \& Wells, K. B. (2008). Interventions in organizational and community context: A framework for building evidence on dissemination and implementation in health services research. Administration and Policy in Mental Health and Mental Health Services Research, 35(1-2), 21-37.

Michie, S., Hyder, N., Walia, A., \& West, R. (2011). Development of a taxonomy of behaviour change techniques used in individual behavioural support for smoking cessation. Addictive Behaviors, 36(4), 315-319.

Moore, G., Audrey, S., Barker, M., Bond, L., Bonell, C., Hardeman, W. et al. (2015). Process evaluation of complex interventions. Complex Interventions in Health: An Overview of Research Methods, (pp. 222). London and New York: Routledge, Taylor \& Francis Group.

National TB Program. (2013). Annual Report Retrieved August 11, 2015, from http://ntp.gov.pk/ uploads/NTP_Annual_Report_2013.pdf.

Piné-Abata, H., McNeill, A., Murray, R., Bitton, A., Rigotti, N., \& Raw, M. (2013). A survey of tobacco dependence treatment services in 121 countries. Addiction, 108(8), 1476-1484. 
Rice, V., \& Stead, L. (2008). Nursing interventions for smoking cessation. Cochrane Database of Systematic Reviews, Issue 1. Art. No.: CD001188. DOI: 10.1002/14651858.CD001188.pub3.

Rimer, B. K., \& Glanz, K. (2005). Theory at a glance: A guide for health promotion practice (2nd ed.). Bethesda, Maryland, National Institutes of Health, National Cancer Institute, p. 57 (NIH Publication No. 05-3896), Retrieved Jan 15, 2016, from http://www.popline.org/node/276257\#sthash.OBRkUUja. dpuf

Rogers, E. M. (2010). Diffusion of innovations (4th ed.). New York: The Free Press, Simon and Schuster Inc.

Rycroft-Malone, J., Kitson, A., Harvey, G., McCormack, B., Seers, K., Titchen, A. et al. (2002). Ingredients for change: Revisiting a conceptual framework. Quality and Safety in Health Care, 11(2), 174-180.

Sereno, A. B., Soares, E. C. C., Lapa e Silva, J. R., Nápoles, A. M., Bialous, S. A., da Costa e Silva, V. L. et al. (2012). Feasibility study of a smoking cessation intervention in directly observed therapy short-course tuberculosis treatment clinics in Rio de Janeiro, Brazil. Rev Panam Salud Publica, 32(6), 451-456.

Siddiqi, K. (2014). Supporting economically disadvantaged tobacco users to help them stop: Time to review priorities?. Addiction, 109(8), 1221-1222.

Siddiqi, K., Khan, A., \& Ahmad, M. (2010). An intervention to stop smoking among patients suspected of TB-evaluation of an integrated approach. BMC Public Health, 10(1), 160.

Siddiqi, K., Khan, A., Ahmad, M., Dogar, O., Kanaan, M., Newell, J. N. et al. (2013). Action to stop smoking in suspected tuberculosis (ASSIST) in Pakistan: A cluster randomized, controlled trial. Annals of Internal Medicine, 158(9), 667-675.

Siddiqi, K., \& Lee, A. C. K. (2009). An integrated approach to treat tobacco addiction in countries with high tuberculosis incidence. Tropical Medicine \& International Health, 14(4), $420-428$.

Siddiquea, B., Islam, M., Bam, T., Satyanarayana, S., Enarson, D., Reid, A. et al. (2013). High quit rate among smokers with tuberculosis in a modified smoking cessation programme in Dhaka, Bangladesh. Public Health Action, 3(3), 243246.

Slama, K., Chiang, C. Y., Enarson, D. A., Hassmiller, K., Fanning, A., Gupta, P. et al. (2007). Tobacco and tuberculosis: A qualitative systematic review and meta-analysis. The International Journal of Tuberculosis and Lung Disease, 11(10), 10491061.

van Zyl Smit, R., Pai, M., Yew, W., Leung, C., Zumla, A., Bateman, E. et al. (2010). Global lung health: The colliding epidemics of tuberculosis, tobacco smoking, HIV and COPD. European Respiratory Journal, 35(1), 27-33.

West, R., Raw, M., McNeill, A., Stead, L., Aveyard, P., Britton, J. et al. (2015). Healthcare interventions to promote and assist tobacco cessation: A review of efficacy, effectiveness and affordability for use in national guideline development. Addiction, 110, 1388-1403.

Whyte, F., \& Kearney, N. (2003). Enhancing the nurse's role in tobacco control. Tobacco Control Factsheets. Retrieved Jan 15, 2016, from https://www.researchgate.net/profile/Nora_ Kearney/publication/266339521_Enhancing_the_nurse\% 27s_role_in_tobacco_control/links/54b664da0cf2e68eb27e 851a.pdf.

World Health Organisation. (2001). WHO Evidence Based Recommendations on the Treatment of Tobacco Dependence. Geneva, WHO.

Wyatt, J., \& Spiegelhalter, D. (1991). Field trials of medical decision-aids: potential problems and solutions. Proceedings of the Annual Symposium on Computer Application in Medical Care, 3-7. 